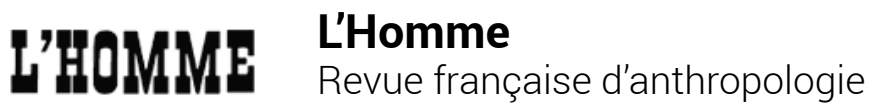

158-159 | avril-septembre 2001

Jazz et anthropologie

\section{Paola Tabet, La Construction sociale de l'inégalité des sexes. Des outils et des corps}

Paris-Montréal, L'Harmattan, 1998, 206 p. (« Bibliothèque du féminisme »)

\section{Patrick Kaplanian}

\section{(2) OpenEdition}

Journals

Édition électronique

URL : http://journals.openedition.org//homme/6470

DOI : $10.4000 /$ lhomme.6470

ISSN : 1953-8103

Éditeur

Éditions de l'EHESS

Édition imprimée

Date de publication : 1 janvier 2001

Pagination : 419-421

ISBN : 2-7132-1386-X

ISSN : 0439-4216

Référence électronique

Patrick Kaplanian, «Paola Tabet, La Construction sociale de l'inégalité des sexes. Des outils et des corps », L'Homme [En ligne], 158-159 | avril-septembre 2001, mis en ligne le 25 mai 2007, consulté le 22 septembre 2020. URL : http://journals.openedition.org//homme/6470 ; DOI : https://doi.org/10.4000/ Ihomme.6470

Ce document a été généré automatiquement le 22 septembre 2020

(c) École des hautes études en sciences sociales 


\section{Paola Tabet, La Construction sociale de l'inégalité des sexes. Des outils et des \\ corps}

Paris-Montréal, L'Harmattan, 1998, 206 p. (« Bibliothèque du

féminisme »)

Patrick Kaplanian

1 SOUS CE TITRE, l'auteur réunit deux textes légèrement remaniés. Le premier, « Les mains, les outils et les armes $»^{1}$, est consacré à la division sexuelle du travail considérée sous un angle nouveau: non pas la séparation des tâches, mais celle des outils. L'auteur s'inscrit d'abord en faux contre les considérations idylliques sur les sociétés « sauvages » où les tâches seraient réparties de façon harmonieuse et complémentaire, complémentarité qui renverrait non pas à une inégalité mais à une différence anatomique, et serait donc « naturelle » (p. 15). Sa thèse est que la division sexuelle du travail «n'est pas neutre, mais orientée et asymétrique [...] il s'agit d'une relation non pas de réciprocité ou de complémentarité mais de domination » (ibid.).

2 Le fil directeur de l'approche, ce sont donc les outils. L'auteur réfute ainsi une autre conception: celle selon laquelle, dans la division équilibrée, harmonieuse et complémentaire, chacun des deux sexes se servirait des outils les plus appropriés à ses propres travaux. Il faut intervertir cette relation: les femmes accomplissent certaines activités, à l'exclusion d'autres, en fonction des outils à utiliser. L'appropriation par les hommes des instruments les plus perfectionnés leur permet d'exercer une domination sur celles-ci. L'auteur parle même de "rapport de classe entre hommes et femmes " (p. 20). Il n'y a d'activités féminines que lorsqu'elles sont accomplies sans outils ou avec des outils simples (p. 21).

3 La méthode est essentiellement statistique ${ }^{2}$, agrémentée de nombreux exemples, d'où une impressionnante bibliographie. La cueillette des produits végétaux est féminine à $80,3 \%$ parce qu'elle se fait à main nue ou avec des outils rudimentaires comme le bâton à fouir qui sert à déterrer des racines. La récolte du miel est masculine à 91,7 \% parce qu'elle nécessite une hache pour couper les troncs et en extraire larves et miel. La 
chasse est à $100 \%$ masculine pour les grands mammifères aquatiques, à 99,4\% pour les gros animaux terrestres, à $98 \%$ quand il s'agit d'oiseaux, à $97,5 \%$ pour la chasse avec pièges de petits mammifères terrestres.

4 Par contre, le ramassage des coquillages, qui s'apparente à la collecte des baies, est le plus souvent féminin. Les femmes peuvent aussi chasser les petits mammifères avec leur bâton à fouir. Lorsqu'elles participent à la chasse, les femmes se spécialisent dans les activités qui n'exigent pas d'arme: crier pour rabattre les animaux, pagayer pendant que l'homme harponne, etc. D'où cette conclusion : sont réservés aux hommes les armes et les outils qui s'y apparentent. Cela est net dans l'agriculture où les hommes fauchent avec la faux ou la faucille tandis que les femmes gerbent. Cela est encore plus net dans la fabrication même des outils où les premiers fabriquent les outils des secondes (taille du bâton à fouir, évidage du canot...) en utilisant haches, couteaux, etc. Pour la même raison, le travail des matières dures (bois, os, métal) est masculin. On pourrait multiplier les exemples, et ils le sont dans ce livre très documenté. Mais se pose une question: les hommes s'approprient-ils les armes en tant qu'outils perfectionnés afin de dominer les femmes, ou bien parce que la guerre est une activité masculine à $100 \%$ ?

5 Paola Tabet élargit alors le débat. D'après elle, toute nouvelle technique plus sophistiquée ou perfectionnée est aussitôt confisquée par les hommes. Ainsi, chez les Baruya, l'introduction des outils en fer ou en acier a permis aux hommes d'améliorer leur travail et de gagner du temps, tandis que les femmes continuent à utiliser le bâton à fouir et les filets pour le transport et travaillent encore plus en raison de l'extension des terres cultivées (p.59). Mais les hommes se sont-ils approprié ces nouvelles techniques parce qu'elles sont plus perfectionnées ou parce qu'il s'agit d'outils-armes? À l'appui de sa thèse, l'auteur donne des exemples d'amélioration d'objets qui ne sont pas des armes. Dès qu'un progrès technique est introduit, les hommes s'attribuent l'instrument, affirme-t-elle. C'est le cas du moulin à vent ou à eau qui a remplacé la meule manuelle. C'est le cas du métier à tisser à pédales ou du tour de potier. Malheureusement, dans cette partie (pp.62-68), les éléments statistiques manquent pour étayer la démonstration. Or, des exemples isolés ne peuvent servir de preuve. L'introduction de l'électroménager en Occident n'a pas vraiment incité les hommes à faire le ménage, la lessive et la vaisselle. Elle a par contre allégé les tâches domestiques des femmes, leur permettant d'y ajouter du travail salarié à l'extérieur. En d'autres termes, il s'est passé pour les femmes en Occident ce qui s'est passé pour les hommes baruya.

6 Le deuxième texte, «Fertilité naturelle et reproduction forcée ${ }^{3}$, traite des méthodes qu'utilisent les hommes pour obliger les femmes à procréer. À la notion de «fécondité naturelle », nos démographes modernes opposent celle de «fertilité dirigée », qui l'est de façon limitative (contraception, avortement). Mais pour nombre de sociétés, y compris dans l'histoire de l'Occident (Paola Tabet donne des exemples florentins et lyonnais), la « direction » est au contraire augmentative. Le but de l'auteur est donc de repérer certains mécanismes tendant à une reproduction imposée, voire forcée (p. 84). Il s'agit non pas d'un contrôle des naissances, mais d'une contrainte à la reproduction.

7 Comment s'y prend-on? Il y a tout d'abord le dressage au coït. L'objectif n'est pas de développer la sexualité des femmes, mais de les soumettre, voire de les «casser ", afin qu'elles acceptent le coït, au moyen de méthodes parfois violentes: viols collectifs, coups... Il y a ensuite la surveillance de la fécondation, de la grossesse et de 
l'accouchement. Certaines sociétés connaissent ou croient connaître le moment fertile et en jouent. D'autres tentent de réduire la période d'allaitement, connue pour être infertile, soit en faisant appel à des nourrices, soit en pratiquant l'infanticide des filles.

Ainsi, dans nombre de sociétés, les femmes sont de véritables machines à reproduire. Et la sexualité dans tout cela? Elle est souvent éliminée pour la femme (cf. le tristement célèbre exemple de l'excision), ou on n'en tient pas compte. Elle peut aussi - le cas est fréquent - être séparée de la reproduction. Deux techniques sont possibles : l'appel à la prostitution («Les courtisanes, nous les avons pour le plaisir, les épouses pour avoir des enfants légitimes ", disait le pseudo-Démosthène) et la séparation en classes d'âge. Les amours entre adolescents sont licites, mais une fois marié on passe aux choses sérieuses, c'est-à-dire à la reproduction. Aussi bien la reproduction est-elle un travail une journée d'allaitement représente, en termes de calories, deux heures de coupe de bois ou neuf heures de marche, etc. (p. 156) -, et un travail exploité.

Une fois de plus, des exemples, fussent-ils nombreux et finement analysés, ne sauraient tenir lieu de preuve, même s'il ne fait pas de doute que la description du sort de la femme que fait Paola Tabet est vraie pour quantité de sociétés. On pourrait néanmoins multiplier les contre-exemples. La sexualité n'est pas toujours génitale (les Baruya pratiquent beaucoup la fellation). L'existence de la prostitution n'implique pas nécessairement la position du pseudo-Démosthène - il suffit de regarder dans notre propre société. La séparation en classes d'âge non plus. Les Trobriandais en sont un bon exemple, d'autant plus qu'ils ne croient pas au rôle du père dans la conception; tout juste aide-t-il à ouvrir la voie. Encore qu'il y ait d'autres moyens. Enfin, certains systèmes tendent à limiter et non à multiplier les naissances. La polyandrie fraternelle himalayenne en est un bon exemple.

\section{NOTES}

1. Cf. L'Homme, 1979, XIX (3-4), nº spéc. : Les catégories de sexe en anthropologie sociale: 5-61.

2. Les statistiques sont celles établies par George P. Murdoch \& Caterina Provost dans « Factors in the Division of Labor by Sex : A Cross-Cultural Analysis », Ethnology, 1973, 12 (2) : 203-225.

3. Cf. L'Arraisonnement des femmes. Essais en anthropologie des sexes. Textes réunis par Nicole-Claude Mathieu. Paris, Éditions de l'École des hautes études en sciences sociales, 1985 (« Cahiers de L'Homme », n. s., XXIV). 


\section{AUTEUR}

\section{PATRICK KAPLANIAN}

Paris. 